\title{
New family of light beams and mirror shapes for future LIGO interferometers
}

\author{
Mihai Bondarescu ${ }^{1, *}$ and Kip S. Thorne ${ }^{2}$ \\ ${ }^{1}$ High Energy Physics, California Institute of Technology, Pasadena, California 91125, USA \\ ${ }^{2}$ Theoretical Astrophysics, California Institute of Technology, Pasadena, California 91125, USA \\ (Received 21 September 2004; revised manuscript received 16 August 2006; published 9 October 2006)
}

Advanced LIGO's present baseline design uses arm cavities with Gaussian light beams supported by spherical mirrors. Because Gaussian beams have large intensity gradients in regions of high intensity, they average somewhat poorly over fluctuating bumps and valleys on the mirror surfaces (thermal noise). Flattopped light beams (mesa beams) are being considered as an alternative because they average over thermal noise more effectively. However, the proposed mesa beams are supported by nearly-flat mirrors, which experience a very serious tilt instability. In this paper we propose an alternative configuration in which mesa-shaped beams are supported by nearly-concentric spheres, which experience only a weak tilt instability. The tilt instability is analyzed for these mirrors in a companion paper by Savov and Vyatchanin. We also propose a one-parameter family of light beams and mirrors in which, as the parameter $\alpha$ varies continuously from 0 to $\pi$, the beams and supporting mirrors get deformed continuously from the nearly-flat-mirrored mesa configuration (FM) at $\alpha=0$, to the nearly-concentricmirrored mesa configuration (CM) at $\alpha=\pi$. The FM and CM configurations at the endpoints are close to optically unstable, and as $\alpha$ moves away from 0 or $\pi$, the optical stability improves.

DOI: 10.1103/PhysRevD.74.082003

PACS numbers: 04.80.Nn, 07.60.Ly, 95.55.Ym

\section{INTRODUCTION}

The initial gravitational-wave detectors in the Laser Interferometric Gravitational wave Observatory (LIGO) are now near design sensitivity and are taking science data [1]. The interferometers will be upgraded to a much more sensitive advanced-LIGO design beginning in early 2011. Until 2003, the baseline design for advanced LIGO used nearly flat but spherical mirrors in its arm cavities. However, in 2003, Sidles and Sigg [2,3] showed that these mirrors experience a strong tilt instability: when the mirrors are tilted symmetrically, the light beam slides across their surfaces to an off-center location and its light pressure then pushes hard to increase the tilt. Sidles and Sigg proposed switching to mirrors that are segments of nearly-concentric spheres (radii of curvature slightly larger than half the cavity length); such mirrors, they showed, can support Gaussian beams of the same (large) radius as the baseline design, while experiencing a much weakened tilt instability. This triggered a change of the baseline design for advanced LIGO to nearly-concentric, spherical mirrors.

Gaussian beams have the serious disadvantage that, because of their steep intensity gradient over most of the beam's area, they average poorly over the fluctuating bumps and valleys on the mirrors' surfaces that are caused by thermal fluctuations (thermoelastic noise). O'Shaughnessy and Thorne [4-6] have proposed improving the averaging and thereby reducing the thermoelastic noise substantially, by replacing the arm cavities' Gaussian beams by flat-topped beams (mesa beams, as Phil Willems has named them), which are supported by nearly-flat,

\footnotetext{
*Electronic address: mihai@tapir.caltech.edu; URL: http://www.tapir.caltech.edu/ mihai
}

Mexican-hat-shaped mirrors. O'Shaughnessy, Strigin and Vyatchanin [6,7] have shown that the substrate thermoelastic noise for mesa beams, at fixed diffraction loss, is 3 times weaker in noise power than for the baseline Gaussian beams, and Agresti [8-10] and Lovelace [11] have shown that substrate Brownian thermal noise and coating thermal noises are about 2 times weaker. Correspondingly, mesabeam interferometers could see significantly farther into the universe than Gaussian-beam interferometers, producing event rates for inspiraling binaries as much as 3 times higher [6]. D'Ambrosio et. al. [6,7,12] have shown that the nearly-flat mesa-beamed mirrors are practical in all respects that could be analyzed theoretically, and research groups at Caltech [13] and Stanford [14] have built prototype optical cavities with mesa beams, and are exploring practical issues experimentally.

Unfortunately, the nearly-flat, Mexican-hat-shaped mirrors ("FM") proposed by O'Shaugnessy and Thorne to support mesa beams (Sec. II below), like the nearly-flat, spherical mirrors of the pre-2003 baseline design, experience a severe tilt instability (Vyatchanin [15]; Savov and Vyatchanin [16]). In this paper, motivated by the SidlesSigg result that, for Gaussian beams and spherical mirrors, the tilt instability is greatly weakened by switching from nearly-flat to nearly-concentric mirrors, we propose (Sec. III) a new, nearly-concentric mirror design ("CM") that supports mesa beams. In a companion paper, Savov and Vyatchanin [16] show that the tilt instability is weaker for these CM mirrors than for any other mirrors thus far considered-FM, nearly-flat spherical, and nearlyconcentric spherical.

In Secs. II and III, we mathematically construct our FM and CM beams and their Mexican-hat mirror shapes by superposing minimal-radius Gaussian beams with optic 


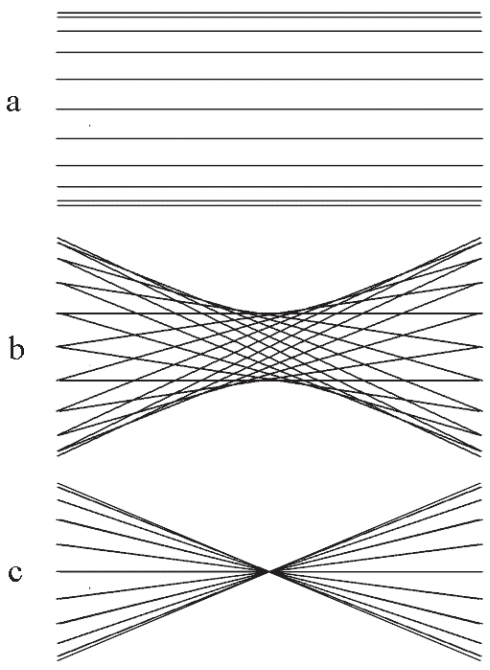

FIG. 1. Optical axes of the families of minimal Gaussian beams used to construct: (a) an FM mesa beam [7], denoted in this paper $\alpha=0$; (c) our new CM mesa beam, denoted $\alpha=\pi$; (b) our new family of hyperboloidal beams, which deform, as $\alpha$ varies from 0 to $\pi$, from a FM beam (a) into a CM beam (c).

axes that are the generators of cylinders (for FM) and of cones (for CM); Fig. 1(a) and 1(c). In Sec. IV we introduce a one-parameter family of "hyperboloidal" light beams and supporting mirrors, computed by superposing minimal Gaussians whose optic axes are the generators of hyperboloids; Fig. 1(b). For each hyperboloidal beam, the hyperboloid's generators (minimal-Gaussian optic axes) have a fixed twist angle $\alpha$. As $\alpha$ is varied continually, the hyperboloidal light beams deform continually from mesa-shaped FM form (at $\alpha=0$, where the hyperboloids are cylinders) to sharply peaked Gaussian form (at $\alpha=\pi / 2$ ), to mesashaped CM form (at $\alpha=\pi$, where the hyperboloids degenerate to cones).

We do not discuss practical aspects of mesa-beam interferometers in this paper. For practical issues (e.g., sensitivity to mirror figure errors and misalignments, creation of mesa beams by driving a Mexican-Hat-mirrored arm cavity with a Gaussian beam, ...), see Refs. $[6,7,13]$.

\section{MESA BEAMS SUPPORTED BY NEARLY-FLAT MIRRORS (FM BEAMS; $\alpha=0$ )}

The mesa beams supported by nearly-flat Mexican-Hat mirrors (FM beams) can be constructed mathematically by a procedure due to O'Shaughnessy and Thorne [5,7]: One superposes minimal Gaussian beams ${ }^{1}$ with their optic axes all parallel to the cavity axis and distributed uniformly over

\footnotetext{
${ }^{1}$ By "minimal Gaussian beam" we mean the fundamental, TEM00 mode of an optical resonator with spherical mirrors, with the mirror radii of curvature adjusted so the Gaussianshaped intensity distributions on the two mirrors are identical and have the minimum possible radii $b$ at the $1 / e$ point of the intensity distribution.
}

a disk with some radius $D$, as shown in Fig. 1(a). Each minimal Gaussian beam (field) is given by

$$
\begin{aligned}
\Psi(\varpi, \zeta)= & \frac{\sqrt{2}}{\sqrt{1+\zeta^{2} / \ell^{2}}} \exp \left\{\frac{-\varpi^{2} / b^{2}}{1+\zeta^{2} / \ell^{2}}+i\left[\frac{\varpi^{2} / b^{2}}{\zeta / \ell+\ell / \zeta}\right.\right. \\
& \left.\left.-\arctan \left(\frac{\zeta}{\ell}\right)+\frac{2 \ell \zeta}{b^{2}}\right]\right\} .
\end{aligned}
$$

Here $\varpi$ is transverse distance from the beam's optic axis; $\zeta$ is distance parallel to the optic axis with $\zeta=0$ at the beam waist; $\ell \equiv L / 2$ is half the length of LIGO's arm cavity $(2 \mathrm{~km})$ and is also equal to the beam's Rayleigh range; $b=$ $\sqrt{\lambda L / 2 \pi}=\sqrt{\lambda \ell / \pi}=2.603 \mathrm{~cm}$ (with $\lambda=1.064 \mu \mathrm{m}$ the light wavelength) is the radius, at the $1 / e$ point of the beam's intensity distribution, at the ends of the cavity, i.e. at $\zeta=\ell$; and $b$ is also the radius, at the $1 / e$ point of the beam's amplitude distribution, at the beam's waist, $\zeta=0$. Note that the last phase factor, $2 \ell \zeta / b^{2}$, is actually $k \zeta$ in disguise, with $k=2 \pi / \lambda$ the light's wave number. We adjust $\lambda$ or $\ell$ slightly so that at $\zeta=\ell$ and $\varpi=0, \Psi$ is real and positive, i.e. the sum of the last two phase factors is a multiple of $2 \pi$. Then in the immediate vicinity of the mirror plane, at $\zeta=\ell+\delta \zeta$ (with $|\delta \zeta| \ll b$ ), the minimal Gaussian has the simple form

$$
\Psi(\varpi, \ell)=\exp \left[\frac{-\varpi^{2}(1-i)}{2 b^{2}}+i k \delta \zeta\right],
$$

with $k=2 \ell / b^{2}$. (Here and throughout this paper we ignore fractional corrections of order $\lambda / b \sim b / \ell \sim$ $10^{-5}$.) The mesa-beam (FM-beam) field, constructed by superposing minimal Gaussians as in Fig. 1(a), is given by (Sec. IIA of [6])

$$
U_{0}(r, z, D)=\int_{\mathcal{C}_{D}} \Psi\left(\sqrt{\left(x-x_{o}\right)^{2}+\left(y-y_{o}\right)^{2}}, z\right) d x_{o} d y_{o}
$$

Here $r=\sqrt{x^{2}+y^{2}}$ is radius from the cavity's central axis, the integral is over Cartesian coordinates $\left(x_{o}, y_{o}\right)$ of the minimal Gaussians' optic axes, and the integral extends over the interior of the disk $\mathcal{C}_{D}$ with radius $D$, i.e. $\sqrt{x_{o}^{2}+y_{o}^{2}} \leq D$. The subscript 0 on $U_{0}$ is the value $\alpha=0$ of the twist angle of the Gaussians' optic axes, when one regards this FM beam from the viewpoint of the hyperboloidal family of beams (Sec. IV below).

By inserting expression (2) with $\delta \zeta=0$ into Eq. (3), we obtain for the FM beam at the mirror plane $z=\ell$,

$$
\begin{aligned}
& U_{0}(r, \ell, D) \\
& \quad=\int_{\mathcal{C}_{D}} \exp \left[\frac{-\left[\left(x-x_{o}\right)^{2}+\left(y-y_{o}\right)^{2}\right][1-i]}{2 b^{2}}\right] d x_{o} d y_{o} .
\end{aligned}
$$

The mirror surface must coincide with a phase front of this mesa beam (FM beam), i.e. it must have a shape $z=$ $\ell+H_{0}(r)$ such that $\arg \left[U_{0}\left(r, \ell+H_{0}, D\right)\right]=$ constant $=$ $\arg \left[U_{0}(0, \ell, D)\right]$. In the vicinity of the mirror the phase of 




FIG. 2. The correction $H_{\alpha}(r)$ to the mirror shape for hyperboloidal beams in a LIGO arm cavity $(L=4 \mathrm{~km})$ with $D=$ $10 \mathrm{~cm}$ and with twist angles $\alpha$ between $\pi / 2$ and $\pi$. For $\alpha=0$, the correction is the negative of that for $\alpha=\pi$; for $\alpha=0.1 \pi$ it is the negative of that for $0.9 \pi$; for any $\alpha$ between 0 and $\pi / 2$, it is the negative of that for $\pi-\alpha$. For $\alpha=\pi$ (the Mexican-hat correction for our new CM mesa beam), $H_{\pi}(r)$ drops to about $-500 \mathrm{~nm}$ (half the wavelength of the light beam) at $r=16 \mathrm{~cm}$ (the mirror's edge). These corrections are added onto the fiducial spheroidal shape $S_{\alpha}(r)$ [Eq. (13)].

each minimal Gaussian varies as $k \delta \zeta=k \delta z$ [Eq. (2)], so $\arg \left[U_{0}\left(r, \ell+H_{0}, D\right)\right]=\arg \left[U_{0}(r, \ell, D)\right]+k H_{0}$, and the shape of the mirror surface must be

$$
H_{0}(r)=k^{-1}\left\{\arg \left[U_{0}(0, \ell, D)-\arg \left[U_{0}(r, \ell, D)\right]\right\} .\right.
$$

By carrying out the integral (4) analytically in one dimension then numerically in the other, and then inserting into Eq. (5), O'Shaughnessy and Thorne find the "Mexicanhat" mirror shape $H(r)$ shown as a solid line in Fig. 2.

To high accuracy, the field $U_{0}$ on the mirror surface differs from that on the plane $z=\ell$ only by the phase factor $e^{i k H_{0}(r)}$, so the intensity distribution on the mirror is the same as at $z=\ell$; i.e., it is

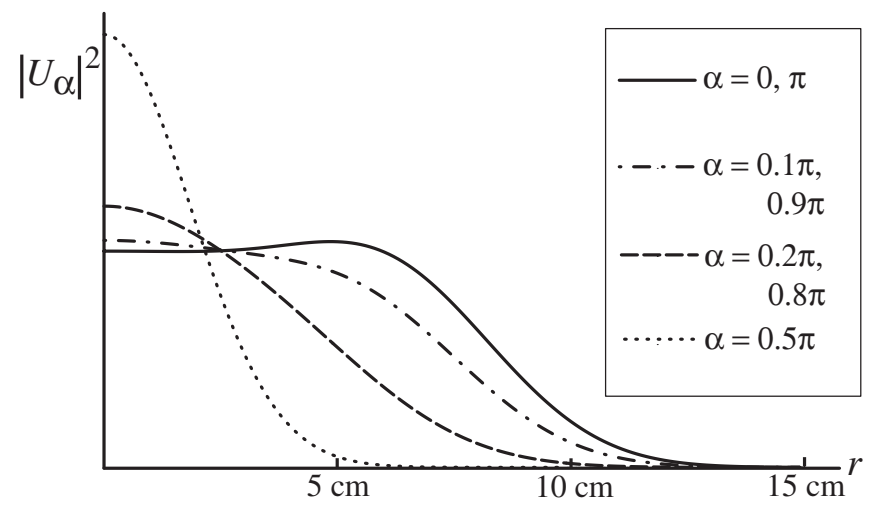

FIG. 3. The light beam's un-normalized intensity $\left|U_{\alpha}\right|^{2}$ as a function of radius $r$ on the mirror, for hyperboloidal beams in a LIGO arm cavity $(L=4 \mathrm{~km})$ with $D=10 \mathrm{~cm}$ and various twist angles $\alpha$. For $\alpha=0$ and $\pi$, the intensity has the mesa shape; for $\alpha=0.5 \pi$ it is a minimal Gaussian.

$$
I_{0}(r) \propto\left|U_{0}(r, \ell, D)\right|^{2} .
$$

This intensity has the mesa shape shown as a solid line in Fig. 3.

\section{MESA BEAMS SUPPORTED BY NEARLY- CONCENTRIC MIRRORS (CM BEAMS; $\alpha=\pi$ )}

Our proposed mesa beams with nearly-concentric, spherical mirrors (CM beams) can be constructed by overlapping minimal Gaussians whose optic axes all pass through the center of the cavity [Fig. 1(c)], and are distributed uniformly inside a cone with angular radius $\Theta=D / \ell$

It should be clear from this construction that the resulting mesa beam will have a beam radius approximately equal to $D$ at the mirrors $(z=\ell)$, and approximately equal to $b$ (the minimal Gaussian radius) at the cavity's center, $z=0$. For advanced LIGO, $D$ is approximately $4 b$ [6], so the waist of this mesa beam will be approximately 4 times narrower than the beam on the mirrors. This contrasts with a mesa beam supported by nearly-flat mirrors (previous section), for which the waist is only slightly narrower than the beam on the mirrors.

Near the mirrors, the phase fronts of this CM beam will be nearly-concentric spheres centered on the point $(r, z)=$ $(0,0)$ through which the Gaussians' optic axes pass, so we shall evaluate the CM field as a function of radius $r$ on this fiducial sphere,

$$
z=S_{\pi}(r) \equiv \sqrt{\ell^{2}-r^{2}} \simeq \ell-r^{2} / 2 \ell .
$$

[Here and below we use a subscript $\pi$ to denote mesa-beam quantities with nearly-concentric mirrors, i.e. CM quantities. This is because the minimal Gaussians used to generate the CM beam have twist angles $\alpha=\pi$; see Sec. IV.] For each minimal Gaussian, this fiducial sphere bends away from the Gaussian's transverse plane by an amount $\delta \zeta=-\varpi^{2} / 2 \ell$, so on this fiducial sphere the Gaussian's phase factor $k \delta \zeta=\left(2 \ell / b^{2}\right) \delta \zeta$ is equal to $-\varpi^{2} / b^{2}$. As a result, the Gaussian field on the fiducial sphere is [cf. Equation (2)]

$$
\begin{aligned}
\Psi\left(\varpi, S_{\pi}\right) & =\exp \left[\frac{-\varpi^{2}(1-i)}{2 b^{2}}+i k \delta \zeta\right] \\
& =\exp \left[\frac{-\varpi^{2}(1+i)}{2 b^{2}}\right] .
\end{aligned}
$$

Correspondingly, these minimal Gaussians superpose, on the fiducial sphere, to produce a CM field given by

$$
\begin{aligned}
& U_{\pi}\left(r, S_{\pi}, D\right) \\
& \quad=\int_{\mathcal{C}_{D}} \exp \left[\frac{-\left[\left(x-x_{o}\right)^{2}+\left(y-y_{o}\right)^{2}\right][1+i]}{2 b^{2}}\right] d x_{o} d y_{o} .
\end{aligned}
$$

Notice that this $U_{\pi}\left(r, S_{\pi}, D\right)$ is the complex conjugate of the FM field $U_{0}(r, \ell, D)$ evaluated on the transverse plane 
$z=\ell$ (the fiducial surface for the case of nearly-flat mirrors); Eq. (4).

As for the nearly-flat (FM) case, the phase of the CM field will vary, with distance $\delta z$ from the fiducial sphere, nearly proportionally to $k \delta z$; and correspondingly, the mirror's surface, $\delta z=H_{\pi}(r)$ (a surface of constant phase), will be given by the analog of Eq. (5):

$$
H_{\pi}(r)=k^{-1}\left\{\arg \left[U_{\pi}\left(0, S_{\pi}, D\right)-\arg \left[U_{\pi}\left(r, S_{\pi}, D\right)\right]\right\} .\right.
$$

Because $U_{\pi}\left(\varpi, S_{\pi}, D\right)$ is the complex conjugate of $U_{0}(\varpi, \ell, D)$, Eqs. (5) and (10) imply that

$$
H_{\pi}(r)=-H_{0}(r) \text {. }
$$

In words: to support mesa beams with the same beam size $D$ on their mirrors, the nearly-concentric mirrors and the nearly-flat mirrors must deviate from precisely concentric spheres $z=S_{\pi}(r)$ and precisely flat planes $z=\ell$ by equal and opposite displacements $\delta z=H(r)$. This fact was discovered in numerical work by one of us (MB) and was later proved numerically in a much wider context by Savov [16] and analytically by Agresti, d'Ambrosio, Chen and Savov [17], before we found the above demonstration.

Because [to the accuracy of our analysis, $O(\lambda / b)$ ] the field $U_{\pi}$ is the same, aside from phase, on the mirror surface as on the fiducial sphere $S_{\pi}$, the light's intensity distribution is the same on the mirror as on $S_{\pi}$ :

$$
I_{\pi}(r) \propto\left|U_{\pi}\left(r, S_{\pi}, D\right)\right|^{2} .
$$

Moreover, because $U_{\pi}\left(r, S_{\pi}, D\right)$ is the complex conjugate of $U_{0}(r, \ell, D)$, they have the same moduli and intensity distributions - i.e., the CM beam has the same mesashaped intensity distribution as the FM beam (solid curve in Fig. 3 below). This fact was discovered in numerical work by one of us (MB) and was later proved numerically in a much wider context by Savov [16] and analytically by Agresti, d'Ambrosio, Chen and Savov [17], before we found the above demonstration.

\section{HYPERBOLOIDAL BEAMS SUPPORTED BY NEARLY SPHEROIDAL MIRRORS}

One can smoothly transform the FM beams into CM beams, and the in-between beams may be interesting for LIGO. In this section, we will focus on one way to make such a transformation.

We will first look at a smooth deformation of the geometric body formed by the optic axes of the minimal Gaussians that are used in constructing the FM and CM beams. For a FM beam, the axes of the minimal Gaussians lie on coaxial cylinders, while for CM beams they lie on coaxial cones. It is well-known that one can smoothly deform a cylinder into a cone as follows. The generators of a cylinder of height $2 \ell$ and radius $r$ [Fig. 1(a)] are lines that join points with cylindrical coordinates $(r, \phi,-\ell)$ on the base circle to points $(r, \phi, \ell)$ on the top circle. The generators of a symmetric cone of height $2 \ell$ and end radii $r$ [Fig. 1(c)] are lines that join points $(r, \phi,-\ell)$ and points $(r, \phi+\pi, \ell)$. A path from the cylinder to the cone is given by a family of hyperboloids generated by lines that join points $(r, \phi,-\ell)$ and points $(r, \phi+\alpha, \ell)$ [Fig. 1(b)]. For $\alpha=0$ one obviously gets the cylinder and for $\alpha=\pi$, the cone.

We therefore propose constructing a new two-parameter family of light beams, and the mirrors that support these beams, using the O'Shaugnessy-Thorne technique of superposing minimal Gaussians. The parameters are $\{\alpha, D\}$, and for given values of $\{\alpha, D\}$ the minimal Gaussians have their optic axes uniformly distributed on the hyperboloid generators that reach from $(r, \phi,-\ell)$ to $(r, \phi+\alpha, \ell)$ [Fig. 1(b)], with $\phi$ running from 0 to $2 \pi$ and $r$ confined to the interior of the $\operatorname{disk} \mathcal{C}_{D}, r \leq D$. For $\alpha=0$ these hyperboloidal beams will be mesa beams with nearly-flat mirrors, i.e. FM beams. For $\alpha=\pi$, they will be mesa beams with nearly-concentric mirrors, i.e. CM beams.

We can construct explicit expressions for the shapes of the mirrors that support these hyperboloidal beams, and expressions for the fields on those mirrors, using the same method as in the FM case (Sec. II) and the CM case (Sec. III): Because the phase of each minimal Gaussian varies nearly proportionally to $k \zeta$, the surface of constant phase at the mirror location will be nearly the same as the "fiducial" surface obtained by cutting off each Gaussian's optic axis at $\zeta=\ell$. One can show that, with the optic axes being generators of hyperboloids, the surface formed by their ends at constant distance $\zeta=\ell$ from the cavity's mid point is the fiducial spheroid

$$
z=S_{\alpha}(r) \equiv \sqrt{l^{2}-r^{2} \sin ^{2}(\alpha / 2)} \simeq \ell-\frac{r^{2} \sin ^{2}(\alpha / 2)}{2 \ell} .
$$

We can compute our hyperboloidal field $U_{\alpha}\left(r, S_{\alpha}, D\right)$ on this fiducial spheroid by superposing our minimal Gaussians with the aid of Fig. 4. In this figure $P$ is the point on the spheroid $S_{\alpha}$ at which we wish to compute the field. The vector $\mathbf{p}$ reaching from the spheroid's center point $O$ (the center of our hyperboloidal field's cross section) to $P$ has Cartesian coordinates $\mathbf{p}=(r, 0, Z)$, where $Z \equiv S_{\alpha}(r)-\ell=-\left(r^{2} / 2 \ell\right) \sin ^{2}(\alpha / 2)$. The optic axis of a minimal Gaussian, over which we will integrate, intersects $S_{\alpha}$ at the point $Q$, which has Cartesian coordinates $\mathbf{q}=\left(r_{o} \cos \phi_{o}, r_{o} \sin \phi_{o}, Z_{o}\right)$, where $Z_{o}=S_{\alpha}\left(r_{o}\right)-$ $\ell=-\left(r_{o}^{2} / 2 \ell\right) \sin ^{2}(\alpha / 2)$. The optic axis of this minimal Gaussian points along the unit vector $\mathbf{n}=\left\{\left(r_{o} / 2 \ell\right) \times\right.$ $\left[\cos \left(\phi_{o}\right)-\cos \left(\phi_{o}-\alpha\right)\right],\left(r_{o} / 2 \ell\right)\left[\sin \left(\phi_{o}\right)-\sin \left(\phi_{o}-\right.\right.$ $\alpha)$ ], 1\}. [Here as elsewhere we neglect corrections of order $r / \ell \sim r_{o} / \ell \sim b / \ell \sim \lambda / b \sim 10^{-5}$.] The vector $\mathbf{s}=$ $\mathbf{p}-(\mathbf{q}+\mathbf{n} \delta \zeta)$ reaches orthogonally from the minimal Gaussian's optic axis to the point $P$. The length of this vector is the radius $\varpi$ of $P$ as measured in cylindrical 


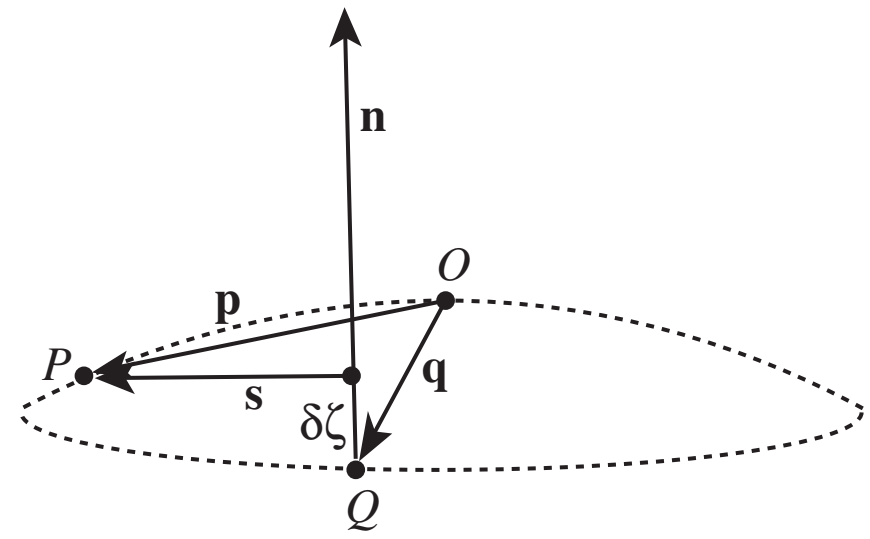

FIG. 4. Geometric construction for computing the hyperboloidal field $U_{\alpha}\left(r, S_{\alpha}, D\right)$ on the fiducial spheroid $S_{\alpha}$ (a segment of which is shown dotted).

coordinates centered on the minimal Gaussian's optic axis,

$$
\varpi=|\mathbf{s}| \simeq|\mathbf{p}-\mathbf{q}|=\sqrt{r^{2}+r_{o}^{2}-2 r r_{o} \cos \phi_{o}},
$$

where the second expression, accurate to $O(\lambda / b)$, can be deduced from the above equations. The distance $\delta \zeta$ along the optic axis $\mathbf{n}$, at which the normal $\mathbf{s}$ intersects the axis, is determined by the orthogonality relation $\mathbf{s} \cdot \mathbf{n}=0$ :

$$
\delta \zeta=\mathbf{n} \cdot(\mathbf{p}-\mathbf{q})=\frac{-1}{2 \ell}\left[\varpi^{2} \sin ^{2}\left(\frac{\alpha}{2}\right)+r r_{o} \sin \alpha \sin \phi_{o}\right] .
$$

The field $U_{\alpha}\left(r, S_{\alpha}, D\right)$ on the spheroid $S_{\alpha}$ is obtained by adding up the minimal Gaussians (2) with $\varpi$ and $\delta \zeta$ given by Eqs. (14) and (15), and with $k=2 \ell / b^{2}$, and by then doing some simple algebra:

$$
\begin{aligned}
U_{\alpha}\left(r, S_{\alpha}, D\right)= & \int_{0}^{D} r_{o} d r_{o} \int_{0}^{2 \pi} d \phi_{o} \exp \left[i \frac{r r_{o}}{b^{2}} \sin \phi_{o} \sin \alpha\right. \\
& \left.-\frac{\left(r^{2}+r_{o}^{2}-2 r r_{o} \cos \phi_{o}\right)}{2 b^{2}}(1-i \cos \alpha)\right] .
\end{aligned}
$$

The radial integral can be carried out analytically yielding an expression involving error functions, and the angular integral can then be done numerically.

The field (16) cannot be sensitive to the chirality of the optic axes' twist, i.e. to the sign of $\alpha$, since it is a scalar complex function of $r: U_{-\alpha}=U_{\alpha}$. This tells us that the relevant range for $\alpha$ is 0 to $\pi$. Replacing $\alpha$ by $\pi-\alpha$ and changing the sign of $\alpha$ is equivalent to complex conjugating $U_{\alpha}$; therefore:

$$
U_{-\alpha}=U_{\alpha} ; \quad U_{\pi-\alpha}=U_{\alpha}^{*} .
$$

For $\alpha=0$, the fiducial spheroid $S_{0}(r)$ is the transverse plane and the field (16) is the FM mesa beam $U_{0}$ [Eq. (4)]. For $\alpha=\pi / 2$, the fiducial spheroid $S_{\pi / 2}$ is a sphere of radius $R=L=2 \ell$ (the distance between the mirrors), and both the radial and the angular integrals can be carried out analytically, giving for the field on that sphere

$$
U_{\pi / 2}=\text { constant } \exp \left[-r^{2} / 2 b^{2}\right]
$$

this is precisely the minimal Gaussian beam [Eq. (2) with $\varpi=r, \quad$ evaluated at $k \delta \zeta=-k r^{2} / 2 R=$ $\left.-\left(2 \ell / b^{2}\right) r^{2} / 4 \ell=-r^{2} / 2 b^{2}\right]$. For $\alpha=\pi$, the fiducial spheroid $S_{\pi}(r)$ is a sphere with radius $\ell=L / 2$ and the field (16) is the CM mesa beam [Eq. (9)]. Thus, as $\alpha$ varies from 0 to $\pi, U_{\alpha}$ deforms continuously from the FM mesa beam $\alpha=0$, through a set of hyperboloidal beams to a minimal Gaussian at $\alpha=\pi / 2$, and on through another set of hyperboloidal beams to the CM mesa beam $\alpha=\pi$.

As for the FM and CM beams, so also for the hyperboloidal beam (16) (and for the same reasons), the mirror's surface must be displaced longitudinally from the fiducial spheroid $z=S_{\alpha}(r)$ by $\delta z=H_{\alpha}(r)$, where

$$
H_{\alpha}(r)=k^{-1}\left\{\arg \left[U_{\alpha}\left(0, S_{\alpha}, D\right)-\arg \left[U_{\alpha}\left(r, S_{\alpha}, D\right)\right]\right\} .\right.
$$

This equation and $U_{\pi-\alpha}=-U_{\alpha}^{*}$ [Eq. (17)] tell us that

$$
H_{\pi-\alpha}(r)=-H_{\alpha}(r) \text {. }
$$

This is a special case of a duality relation discovered

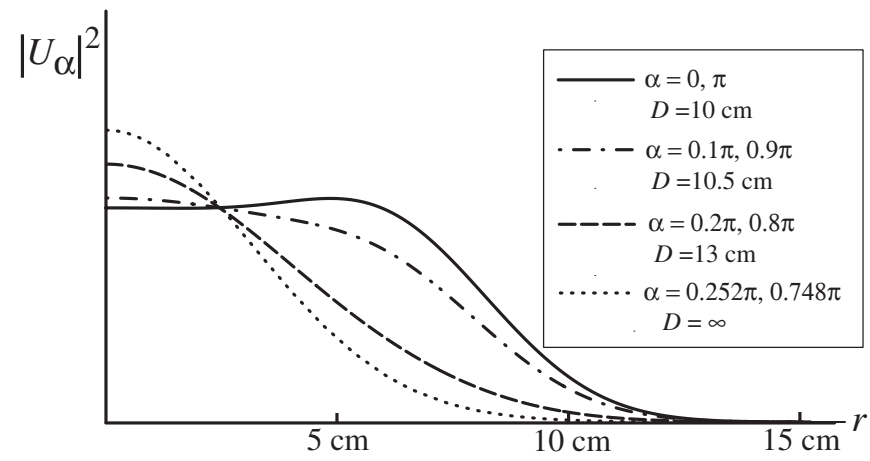

FIG. 5. The light beam's un-normalized intensity $\left|U_{\alpha}\right|^{2}$ as a function of radius $r$ on the mirror, for hyperboloidal beams in a LIGO arm cavity $(L=4 \mathrm{~km})$ with fixed diffraction losses: $2.68 \mathrm{ppm}$ in the clipping approximation, assuming mirror radii of $16 \mathrm{~cm}$. The mesa beam $(\alpha=0)$ has $D=10 \mathrm{~cm}$ and is identical to that of Fig. 3. For $\alpha=0.1 \pi$, to keep the diffraction losses at $2.68 \mathrm{ppm}, D$ has been increased to $10.5 \mathrm{~cm}$ and the physical beam diameter is, correspondingly, a bit larger than in Fig. 3. For $\alpha=0.2 \pi, D$ has been increased to $D=13.0 \mathrm{~cm}$; for $\alpha=0.252 \pi$, it has been increased to $D=\infty$, producing a beam shape that is Gaussian to the accuracy of our numerical computations, but is substantially larger than the minimal Gaussian of Fig. 3 and is approximately the same as the baseline design for advanced LIGO. (Our numerical computations suggest that for $D=\infty$ and all $\alpha \neq 0$ or $\pi$, the hyperboloidal beam is Gaussian, with width varying from minimal, $\sigma=b=\sqrt{\lambda L / 2 \pi}$ at $\alpha=$ $\pi / 2$ to $\sigma \rightarrow \infty$ as $\alpha \rightarrow 0$ or $\pi$, but we have not been able to prove this analytically.) 
numerically by Savov and Vyatchanin [16] and proved analytically by Agresti, d'Ambrosio, Chen and Savov [17]. Figure 2 shows these mirror shape corrections for various $\alpha$ 's. The light intensity on the mirrors is given by the obvious analog of Eqs. (6) and (12):

$$
I_{\alpha}(r) \propto\left|U_{\alpha}\left(r, S_{\alpha}, D\right)\right|^{2} .
$$

This equation and $U_{\pi-\alpha}=-U_{\alpha}^{*}$ [Eq. (17)] tell us that

$$
I_{\pi-\alpha}(r)=I_{\alpha}(r) \text {. }
$$

This is another special case of the duality relation discovered by Savov and Vyatchanin [16], and proved analytically by Agresti, d'Ambrosio, Chen and Savov [17].

The intensity distribution (21) is shown in Fig. 3, for fixed $D=10 \mathrm{~cm}$ and various $\alpha$. To minimize thermal noises, one wants the largest beam compatible with acceptable diffraction losses. In this spirit, in Fig. 5 we compare hyperboloidal beams with fixed diffraction losses (as computed in the clipping approximation) rather than with fixed D.

\section{CONCLUSIONS}

For twist angles $\alpha$ near 0 and $\pi$, the hyperboloidal beams introduced in this paper have the flat-top form needed to reduce thermoelastic noise in LIGO. The radius of the flat top is largest for $\alpha=0$ and $\alpha=\pi$ (the FM and
CM mesa beams) and smallest for $\alpha=\pi / 2$ (the minimal Gaussian).

Because the mirrors are most nearly flat or concentric for the mesa configurations, $\alpha=0$ or $\pi$, those configurations are most nearly optically unstable. (Near instability goes hand in hand with large beams on the mirrors, which are needed to control thermoelastic noise.)

The results of Savov and Vyatchanin [17] suggest that the tilt instability is smallest for $\alpha=\pi$ and worst for $\alpha=$ 0 .

These considerations suggest that the optimal configuration for advanced LIGO will be near $\alpha=\pi$, but whether the optimum is precisely at $\alpha=\pi$ (the CM configuration) or at some modestly smaller $\alpha$ will depend on practical and thermal-noise considerations not examined in this paper.

\section{ACKNOWLEDGMENTS}

We thank Pavlin Savov, Juri Agresti, Erika D'Ambrosio, Yanbei Chen, Geoffery Lovelace, and Poghos Kazarian for useful discussions and advice. M.B. thanks Manuela Campanelli and the University of Texas at Brownsville, and Ed Seidel, Gabrielle Allen and the Center for Computation and Technology at Louisiana State University for helpful discussions and travel support during this research. This research was supported in part by NSF Grants No. PHY-0099568 and PHY-0601459.
[1] LIGO, http://www.ligo.caltech.edu/.

[2] J. Sidles and D. Sigg, LIGO Report No. T0301320-00, 2003, http://www.ligo.caltech.edu/docs/T/T030120-00. pdf.

[3] J. Sidles and D. Sigg, Phys. Lett. A 354, 167 (2006).

[4] K.S. Thorne, LIGO Report No. G000068-00-D, 2000, http://www.ligo.caltech.edu/docs/G/G000068-00.pdf.

[5] E. D'Ambrosio, R. O'Shaughnessy, and K. S. Thorne, LIGO Report No. G000223-00-D, 2000, http://www.ligo. caltech.edu/docs/G/G000223-00.pdf.

[6] E. D’Ambrosio, R. O'Shaugnessy, S. Strigin, K. S. Thorne, and S. Vyatchanin, gr-qc/0409075.

[7] R. O'Shaunessy, S. Strigin, and S. Vyatchanin, gr-qc/ 0409050.

[8] J. Agresti, LIGO Technical Report No. T040225-00-R (unpublished); http://www.ligo.caltech.edu/docs/T/ T040225-00.pdf.
[9] J. Agresi and R. DeSalvo, Report No. LIGO-G050041-00$\mathrm{Z}$ (unpublished); http://www.ligo.caltech.edu/docs/G/ G050041-00.

[10] J. Agresti (unpublished).

[11] G. Lovelace (unpublished).

[12] E. D’Ambrosio, Phys. Rev. D 67, 102004 (2003).

[13] J. Agresti et al., LIGO Report No. G050522-00-Z, 2005, http://www.ligo.caltech.edu/docs/G/G050522-00/ G050522-00.pdf.

[14] P. T. Beyersdorf, S. Zappe, M. M. Fejer, and M. Burkhardt (unpublished).

[15] S. Vyatchanin, LIGO Report Number T030272-00, 2003, http://www.ligo.caltech.edu/docs/T/T030272-00.pdf.

[16] S. Vyatchanin and P. Savov, Phys. Rev. D 74, 082002 (2006).

[17] P. Savov, E. D’Ambrosio, Y. Chen, and J. Agresti, gr-qc/ 0511062 . 